in vivo $34: 2981-2989(2020)$

doi:10.21873/invivo.12129

\title{
Prognostic Utility of Apical Lymph Node Metastasis in Patients With Left-sided Colorectal Cancer
}

\author{
LIMING WANG, YASUMITSU HIRANO, GREGORY HENG, TOSHIMASA ISHII, \\ HIROKA KONDO, KIYOKA HARA, NAO OBARA, MASAHIRO ASARI and SHIGEKI YAMAGUCHI
}

Division of Gastroenterological Surgery, Saitama Medical University International Medical Center, Hidaka, Japan

\begin{abstract}
Background: Unlike the tumor nodes metastasis (TNM) lymph node classification, based solely on counts of nodal metastases, the Japanese system of classifying colorectal carcinoma (CRC) focuses on regional lymph node spread. In this study, we explored the prognostic utility of inferior mesenteric artery (IMA) apical lymph node (APN) metastasis. Patients and Methods: This was a retrospective study of patients with stage III left-sided CRC. All enrollees were subjected to D3 resection between April 2007 and December 2016 at the International Medical Center of Saitama Medical University and then stratified by histologic presence (APN+ group) or absence (APN- group) of tumor in APNs examined postoperatively. Ultimately, propensity score matching was invoked (1:2) and COX regression analysis was conducted, determining group rates of relapse-free survival (RFS) and cancer-specific survival (CSS). Results: A total of 498 patients were studied, grouped as APN+ $(19 / 498,3.8 \%)$ or APN(479/498, 96.2\%). Prior to matching, the APN+ (vs. APN-) group showed significantly more lymphatic involvement $(73.7 \%$ vs. 47.8\%; $p=0.023)$, deep (T3/T4) tumor infiltration $(100 \%$ vs. $78.9 \% ; p=0.024)$, and nodal metastasis (N2: $84.2 \%$ vs. $27.6 \% ; p<0.001)$. In addition, para-aortic nodal recurrences were significantly increased $(15.7 \%$ vs. $2.0 \% ; p<0.001)$, conferring worse RFS $(p<0.001)$ and CSS $(p=0.014)$ rates. Once baseline factors were matched, the two groups appeared similar in RFS $(p=0.415)$ and CSS $(p=0.649)$. Multivariate regression analysis indicated that elevated carcinoembryonic antigen (CEA) level and deep tumor infiltration were independent risk factors for RFS, whereas postoperative
\end{abstract}

This article is freely accessible online.

Correspondence to: Liming Wang, MD, Ph.D., Division of Gastroenterological Surgery, Saitama Medical University International Medical Center, Hidaka, Saitama, Japan. Tel: +8142 9844111, Fax: +81 429840054, e-mail: wuminami@hotmail.com

Key Words: Apical lymph nodes, left-sided colorectal cancer, propensity score matching. complications and tumor-positive node counts were independent risk factors for CSS. APN+ status was not a significant risk factor for RFS or CSS. Conclusion: APN positivity may thus constitute a regional rather than systemic manifestation. The TNM staging based on the number of metastatic lymph nodes seems to be more reasonable than the regional lymph node classification method.

Metastasis of colorectal cancer (CRC) to lymph nodes is an important predictor of patient survival and indicator for the need of postoperative adjuvant chemotherapy $(1,2)$. The Japanese Classification of Colorectal, Appendiceal, and Anal Carcinoma is based on lymph node distribution $(2,3)$, whereas guidelines of the American Joint Conference on Cancer (AJCC) and the International Union Against Cancer (UICC) tumor nodes metastasis (TNM) Classification of Malignant Tumours recommend a 12-node sampling at minimum, focusing more on absolute numbers of nodal metastases. For example, a single apical lymph node (APN) qualifying as N1a by TNM standards warrants a N3 designation in the Japanese system of classification (4).

In recent years, the role of APNs has fueled considerable controversy. It has been argued that the anatomic distribution of nodal metastases may supplement the current TNM staging system (5), and that APNs may be the gateway to systemic metastasis (6). Some publications have championed APN positivity as an independent prognostic risk factor (4, 7-10) to the dismay of other investigators $(1,11)$. Such debate is largely due to baseline differences in patient groups.

Propensity score matching (PSM) has been widely adopted in clinical research because it helps reduce the baseline bias of comparator groups $(12,13)$. This study was undertaken to explore the prognostic utility of inferior mesenteric artery (IMA) APN metastasis in patients with left-sided CRC.

\section{Patients and Methods}

As candidates for this retrospective analysis, a total of 2,457 patients underwent surgical resections of left-sided CRC at the International Medical Center of Saitama Medical University between April 2007 and December 2016. Exclusion criteria were as follows: 1) recurrent 


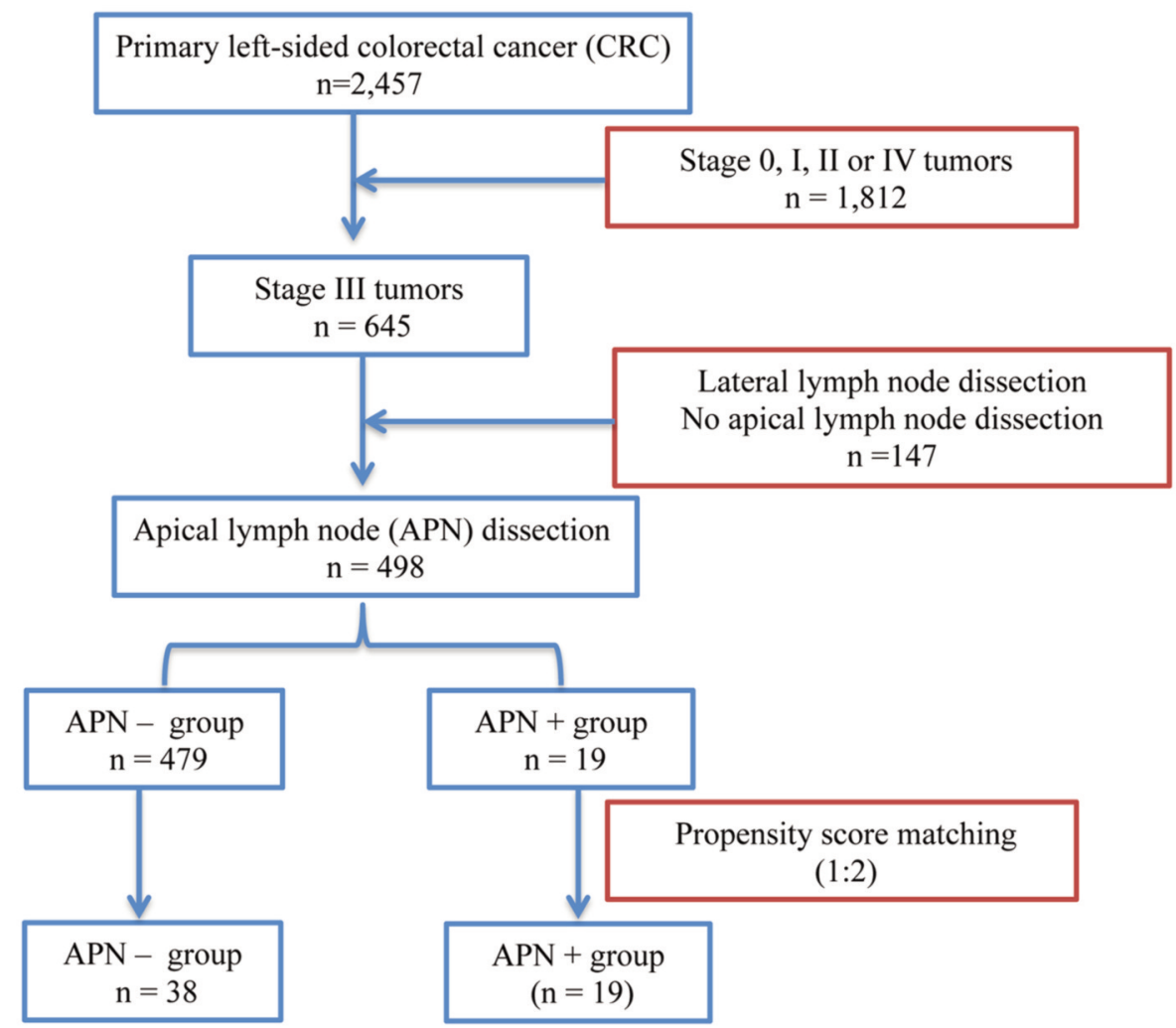

Figure 1. Schematic of patient allocation in primary left-sided colorectal cancer. APN-, Tumor-negative apical lymph nodes; APN+, tumor-positive apical lymph nodes.

CRC, 2) multiple malignant tumors, 3) non-resectability, or 4) distant metastasis. Nodal metastases were detected postoperatively in 645 patients. Patients with low-lying rectal cancers involving lateral lymph nodes and those unfit for $\mathrm{D} 3$ resection were then disqualified, leaving 498 patients for study (Figure 1).

We used a medial to lateral approach along the root of IMA (cephalad side) for D3 lymph node dissection. After location of the abdominal aorta, both hypogastric nerves were exposed to ensure their preservation (Figure 2). Nodal metastases were identified systemetically, harvesting pericolorectal lymph nodes $(231,241$, 251) (231: pericolic lymph node of descending colon; 241 : pericolic lymph node of sigmoid colon; 251: perirectal lymph node), inferior mesenteric trunk nodes $(232,242,252)$ (232: intermediate lymph node of descending colon; 242: intermediate lymph node of sigmoid colon; 252: intermediate lymph node of rectal), and IMA APNs (253) for prognostic analysis. Ultimately, all patients were grouped by APN status as tumor-positive (APN+) or tumor-negative (APN-), comparing baseline characteristics of the two groups.

Propensity score matching (PSM) was invoked to offset the differences between the two groups. Age, sex, preoperative carcinoembryonic antigen (CEA) level, duplicate cancers, multiple cancers, tumor location, surgical method, depth of tumor infiltration, nodal metastasis, lymphatic involvement, perineural infiltration, vascular invasion, pathologic staging, and more were incorporated in PSM. After matching, COX regression analysis was conducted to compare relapse-free survival (RFS) and cancer-specific survival (CSS) rates.

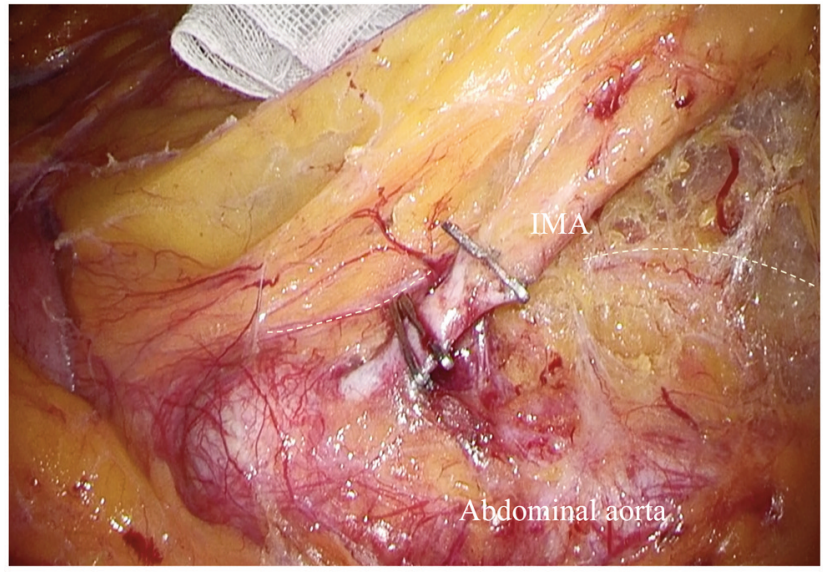

Figure 2. Dissection of inferior mesenteric artery (IMA) apical lymph nodes (APNs). Ventral aspect of abdominal aorta borders on lymph nodes dissected at IMA; white dotted line marking left hypogastric nerve segment.

All computations were driven by standard software (SPSS v22 for Mac; IBM Corp, Armonk, NY, USA). Chi-square test and Fisher's exact test were applied to assess differences in categorical variables, using Kaplan-Meier method to plot $t$-test values. 


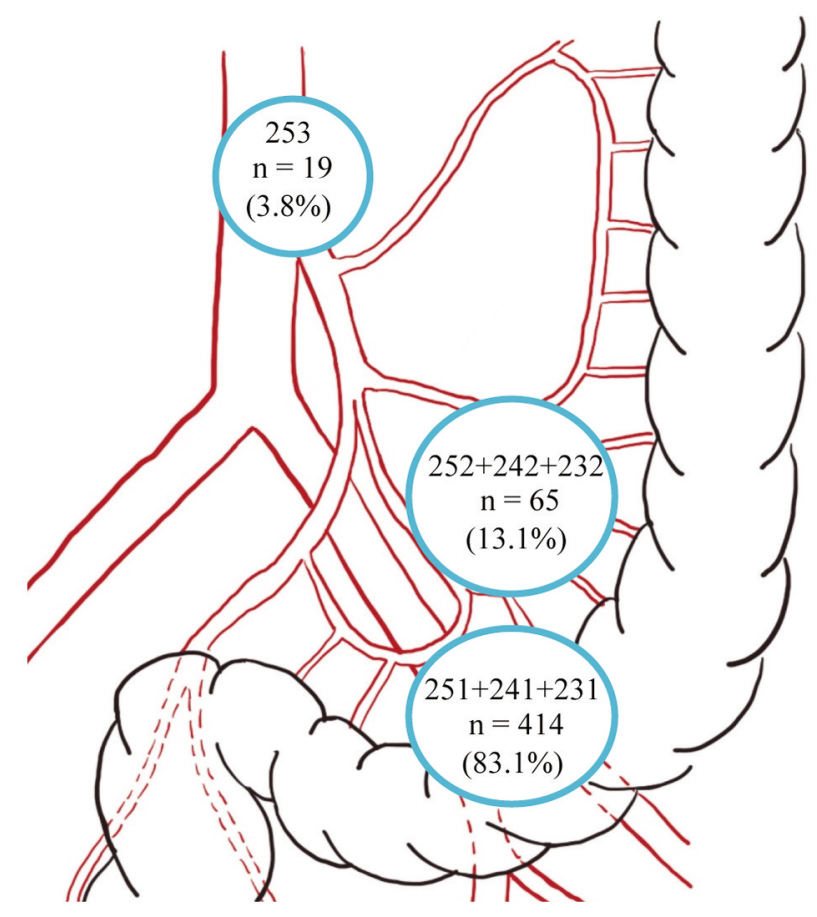

Figure 3. Distribution of lymphatic metastasis in patients subjected to D3 nodal dissection. 231, Pericolic lymph node of descending colon; 241 , pericolic lymph node of sigmoid colon; 251, perirectal lymph node; 232 , intermediate lymph node of descending colon; 242 , intermediate lymph node of sigmoid colon; 252, intermediate lymph node of rectal; 253, apical lymph node of IMA.

\section{Results}

Of the 498 patients studied, the APN+ group accounted for $19(3.8 \%)$ patients. In the APN- group (479/498, 96.2\%), metastases of inferior mesenteric trunk $(65 / 498,13.1 \%)$ $(232,242,252)$ and pericolorectal $(414 / 479,83.1 \%)(231$, $241,251)$ lymph nodes were still encountered (Figure 3). RFS $(p<0.001)$ and OS $(p=0.001)$ significantly declined as nodal metastasis approached the abdominal aorta (Figure 4A and $\mathrm{B})$.

Clinical and pathological data of the two groups were subsequently analyzed. The APN+ (vs. APN-) group showed significantly more lymphatic invasion $(73.7 \%$ vs. $47.8 \%$ $p=0.023$ ), deep tumor infiltration (T3/T4: $100 \%$ vs. $78.9 \%$; $p=0.024)$, and nodal metastasis ( 2 : $84.2 \%$ vs. $27.6 \%$, $p<0.001)$. There were also significantly more open surgeries (26.3\% vs. $9.8 \% ; p=0.038)$ by comparison (Table I), and prior to PSM, RFS $(p<0.001)$ and CSS $(p=0.014)$ rates were significantly lower (Figure $5 \mathrm{~A}$ and $\mathrm{B}$ ).

To accurately depict the effect of various parameters on patient prognosis, Cox regression analysis was performed in advance of PSM. Significant independent predictors of RFS
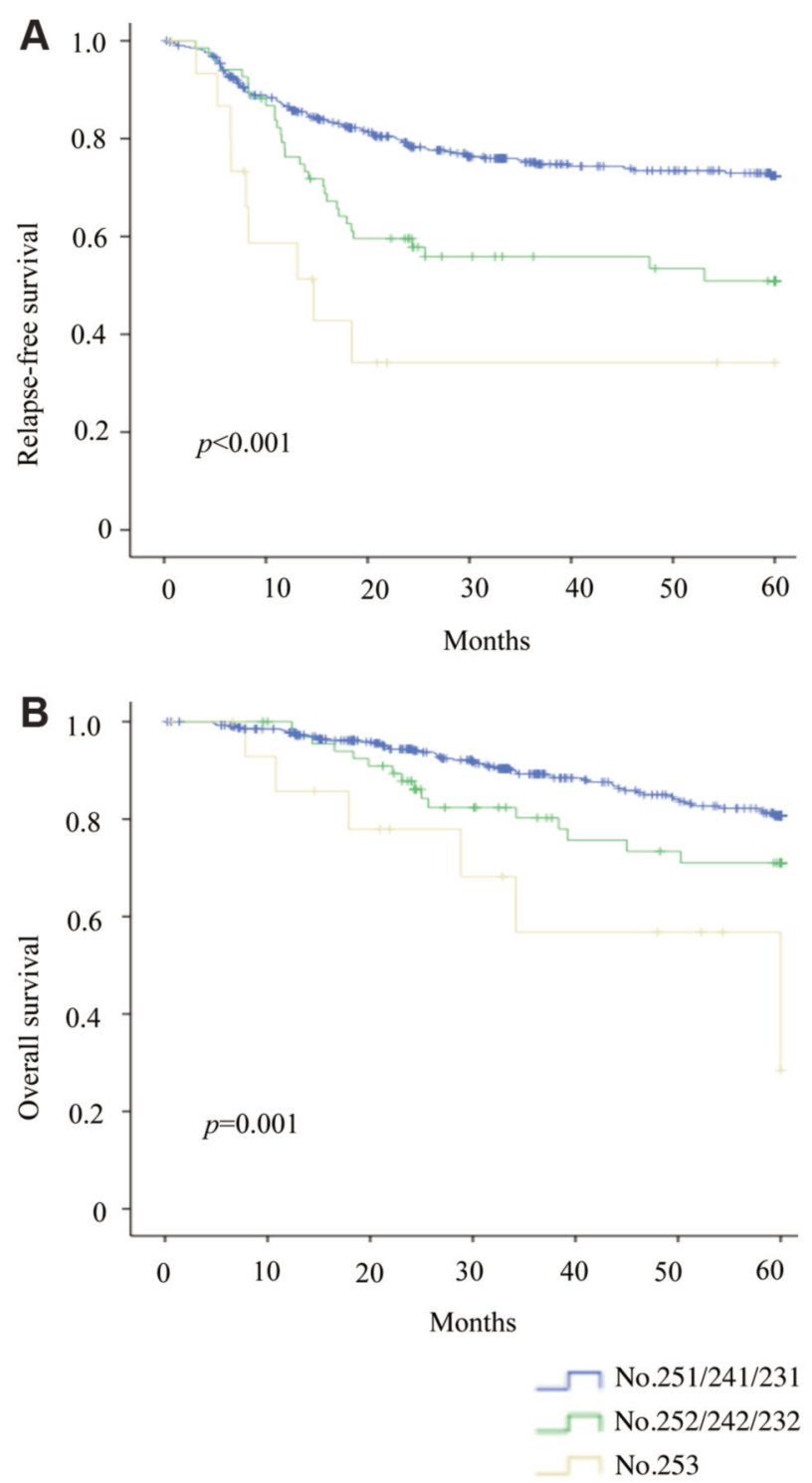

Figure 4. Outcomes of patients with stage III colorectal cancer (CRC): Relapse-free survival $(A)$ and overall survival $(B)$.

and CSS were CEA level [RFS, Hazard ratio $(\mathrm{HR})=0.669$ (95\% confidence interval $(\mathrm{CI})=0.472-0.948 ; p=0.024)$; CSS, $\mathrm{HR}=0.505(95 \% \mathrm{CI}=0.293-0.87 ; p=0.014)]$; tumor-positive node count [RFS, HR=1.112 (95\% CI=1.042-1.187; $p=0.001)$; CSS, HR=1.181 (95\% CI=1.076-1.297; $p<0.001)]$; and depth of tumor infiltration [RFS, T3/T4 HR=0.348 $(95 \%$ $\mathrm{CI}=0.172-0.706 ; p=0.003)$; $\mathrm{CSS}, \mathrm{HR}=0.215$ (95\% CI=0.049$0.946 ; p=0.042)]$. APN+ status was not a significant risk factor for RFS $(p=0.5)$ or CSS ( $p=0.637$ ) (Table II).

PSM was performed at a ratio of $1: 2$, comparing 19 APN+ patients with 38 matched APN- counterparts. Baseline characteristics of the two groups were similar 
in vivo $34: 2981-2989(2020)$

Table I. Clinicopathological characteristics of patients with stage III left-sided colorectal cancer.

\begin{tabular}{|c|c|c|c|c|c|c|}
\hline \multirow[t]{2}{*}{ Parameters } & \multicolumn{3}{|c|}{ Before matching } & \multicolumn{3}{|c|}{ After matching } \\
\hline & APN- & $\mathrm{APN}+$ & $p$-Value & APN- & $\mathrm{APN}+$ & $p$-Value \\
\hline Gender (Total $n=)$ & 479 & 19 & & 38 & 19 & \\
\hline Male & $307(64.1)$ & $10(52.6)$ & & & $21(55.3)$ & $10(52.6)$ \\
\hline Female & $172(35.9)$ & $9(47.4)$ & N.S. & $17(44.7)$ & $9(47.4)$ & N.S. \\
\hline Age (year) & $65.1 \pm 0.47$ & $63.8 \pm 2.15$ & N.S. & $64.4 \pm 1.48$ & $63.8 \pm 2.15$ & N.S. \\
\hline \multicolumn{7}{|l|}{ CEA (ng/ml) } \\
\hline$\geq 5$ & $175(36.5)$ & $9(47.4)$ & & $15(39.5)$ & $9(47.4)$ & \\
\hline$<5$ & $304(63.5)$ & $10(52.6)$ & N.S. & $23(60.5)$ & $10(52.6)$ & N.S. \\
\hline \multicolumn{7}{|l|}{ Duplicate cancer } \\
\hline No & $425(88.7)$ & $17(89.5)$ & & $33(86.8)$ & $17(89.5)$ & \\
\hline Yes & $54(11.3)$ & $2(10.5)$ & N.S. & $5(13.2)$ & $2(10.5)$ & N.S. \\
\hline \multicolumn{7}{|l|}{ Multiple cancers } \\
\hline No & $441(92.1)$ & $17(89.5)$ & & $36(94.7)$ & $17(89.5)$ & \\
\hline Yes & $38(7.9)$ & $2(10.5)$ & N.S. & $2(5.3)$ & $2(10.5)$ & N.S. \\
\hline Laparoscopy & $432(90.2)$ & $14(73.7)$ & & $35(92.1)$ & $14(73.7)$ & \\
\hline Open resection & $47(9.8)$ & $5(26.3)$ & 0.038 & $3(7.9)$ & $5(26.3)$ & N.S. \\
\hline \multicolumn{7}{|l|}{ Tumour location } \\
\hline Descending colon & $30(6.3)$ & $0(0.0)$ & & $0(0.0)$ & $0(0.0)$ & \\
\hline Sigmoid colon & 161 (33.6) & $6(31.6)$ & & $9(23.7)$ & $6(31.6)$ & \\
\hline $\mathrm{RS} / \mathrm{Ra}$ & $171(35.7)$ & $6(31.6)$ & & $15(39.5)$ & $6(31.6)$ & \\
\hline $\mathrm{Rb}$ & $117(35.7)$ & $7(36.8)$ & N.S. & $14(36.8)$ & $7(36.8)$ & N.S. \\
\hline \multicolumn{7}{|l|}{ Tumor histotype } \\
\hline $\mathrm{ADC}$ & $455(95.0)$ & 18 (94.7) & & $37(97.4)$ & $18(94.7)$ & \\
\hline MUC, SRC, Poor & $24(5.0)$ & $1(5.3)$ & N.S. & $1(2.6)$ & $1(5.3)$ & N.S. \\
\hline \multicolumn{7}{|l|}{ Gross appearance } \\
\hline Protruding & $63(13.2)$ & $0(0.0)$ & & $0(0.0)$ & $0(0.0)$ & \\
\hline Infiltrating/ulcerative & $416(86.8)$ & $19(100.0)$ & N.S. & $38(100.0)$ & $19(100.0)$ & N.S. \\
\hline \multicolumn{7}{|l|}{ Perineural infiltration } \\
\hline No & $118(24.6)$ & $4(21.1)$ & & $6(15.8)$ & $4(21.1)$ & \\
\hline Yes & $361(75.4)$ & $15(78.9)$ & N.S. & $32(84.2)$ & $15(78.9)$ & N.S. \\
\hline \multicolumn{7}{|l|}{ lymphatic involvement } \\
\hline No & $250(52.2)$ & $5(26.3)$ & & $10(26.3)$ & $5(26.3)$ & \\
\hline Yes & $229(47.8)$ & $14(73.7)$ & 0.023 & $28(73.1)$ & $14(73.7)$ & N.S. \\
\hline \multicolumn{7}{|l|}{ Vascular invasion } \\
\hline No & $124(25.9)$ & $4(21.1)$ & & $3(7.9)$ & $4(21.1)$ & \\
\hline Yes & $355(74.1)$ & $15(78.9)$ & N.S. & $35(92.1)$ & $15(78.9)$ & N.S. \\
\hline \multicolumn{7}{|l|}{ Infiltrative depth } \\
\hline $\mathrm{T} 1-2$ & $101(21.1)$ & $0(0.0)$ & & $0(0.0)$ & $0(0.0)$ & \\
\hline T3-4 & $378(78.9)$ & $19(100.0)$ & 0.024 & $38(100.0)$ & $19(100.0)$ & N.S. \\
\hline \multicolumn{7}{|l|}{ Nodal metastasis } \\
\hline N1 & $347(72.4)$ & $3(15.8)$ & & $6(15.8)$ & $3(15.8)$ & \\
\hline N2 & $132(27.6)$ & $16(84.2)$ & $<0.001$ & $32(84.2)$ & $16(84.2)$ & N.S. \\
\hline
\end{tabular}

ADC, Adenocarcinoma; CEA, carcinoembryonic antigen; MUC, mucinous adenocarcinoma; Poor, poorly differentiated adenocarcinoma; SRC, signet-ring cell carcinoma.

(Table I). In Cox regression analysis, CEA level (HR=0.446, $95 \% \mathrm{CI}=0.157-1.269 ; p=0.013)$ and depth of tumor infiltration $(\mathrm{T} 3 / \mathrm{T} 4 \mathrm{HR}=8.011,95 \% \quad \mathrm{CI}=1.589-40.393$; $p=0.012)$ remained independent risk factors for RFS. Age $(\mathrm{HR}=2.149,95 \% \mathrm{CI}=1.249-3.696 ; p=0.006)$, postoperative complications $(\mathrm{HR}=601.2 ; 95 \% \mathrm{CI}=3.219-11227 ; p=0.016)$, CEA level $(\mathrm{HR}=0.460 ; 95 \% \mathrm{CI}=0.02-10.355 ; p=0.01)$, and tumor-positive node count $(\mathrm{HR}=3.592 ; 95 \% \mathrm{CI}=1.186$ $10.876 ; p=0.024$ ) emerged as independent risk factors for
CSS. Again, APN metastasis was not a significant risk factor for RFS $(p=0.379)$ or CSS $(p=0.151)$ (Table III). Of note, RFS $(p=0.415)$ and CSS $(p=0.649)$ did not differ significantly (Figures 6A, 6B).

Finally, we explored potential differences in perioperative outcomes through comparative analysis. Before PSM, the APN+ ( $v s$. APN-) group showed significantly more bleeding (134.53 $\pm 38.47 \mathrm{ml} v s .48 .43 \pm 5.95 \mathrm{ml} ; p=0.006)$ and number of nodal metastases $(9.58 \pm 1.64$ vs. 2.98 \pm 0.12 ; 

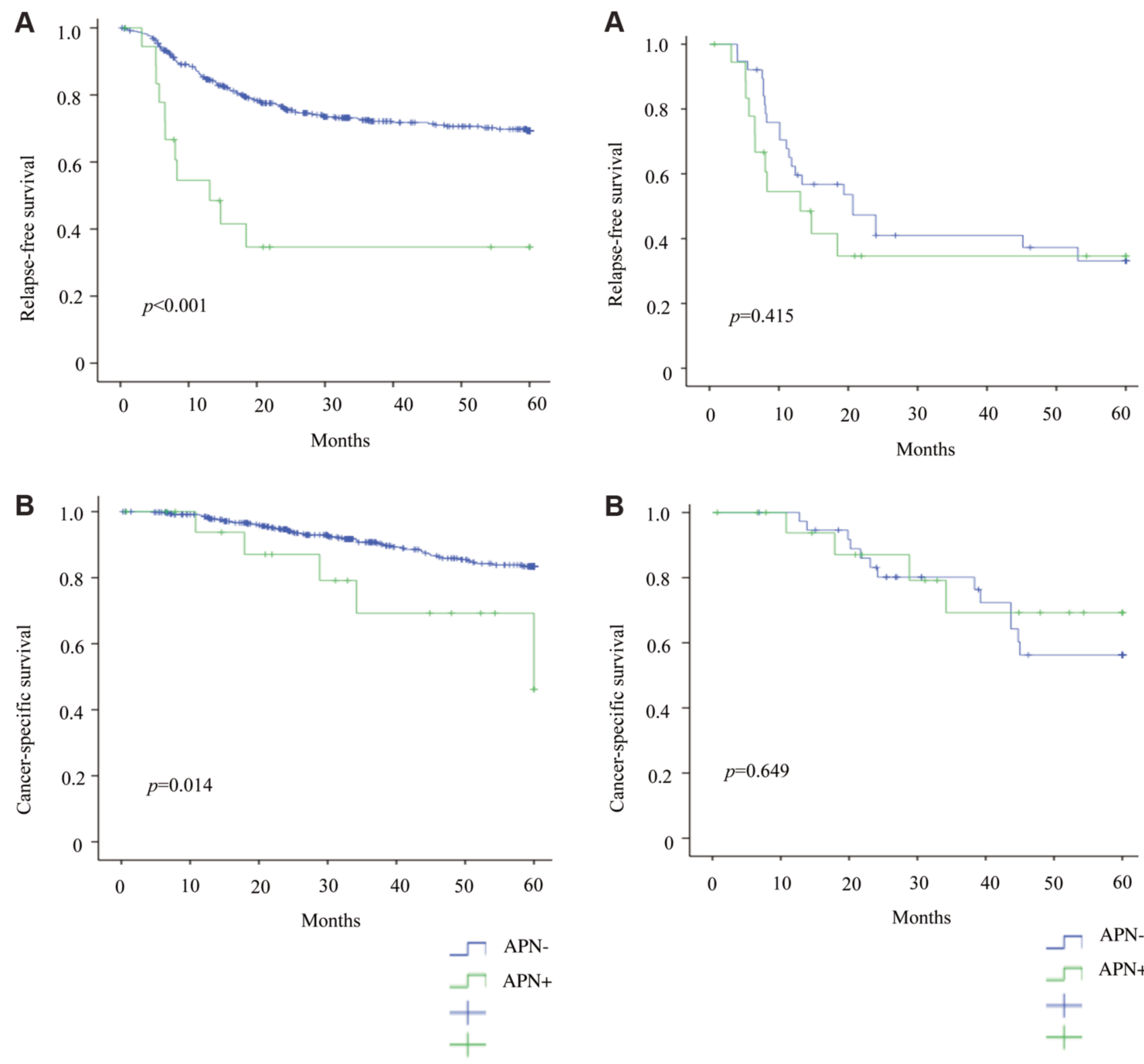

Figure 5. Patient outcomes prior to matching: (A) Relapse-free survival and (B) cancer-specific survival.

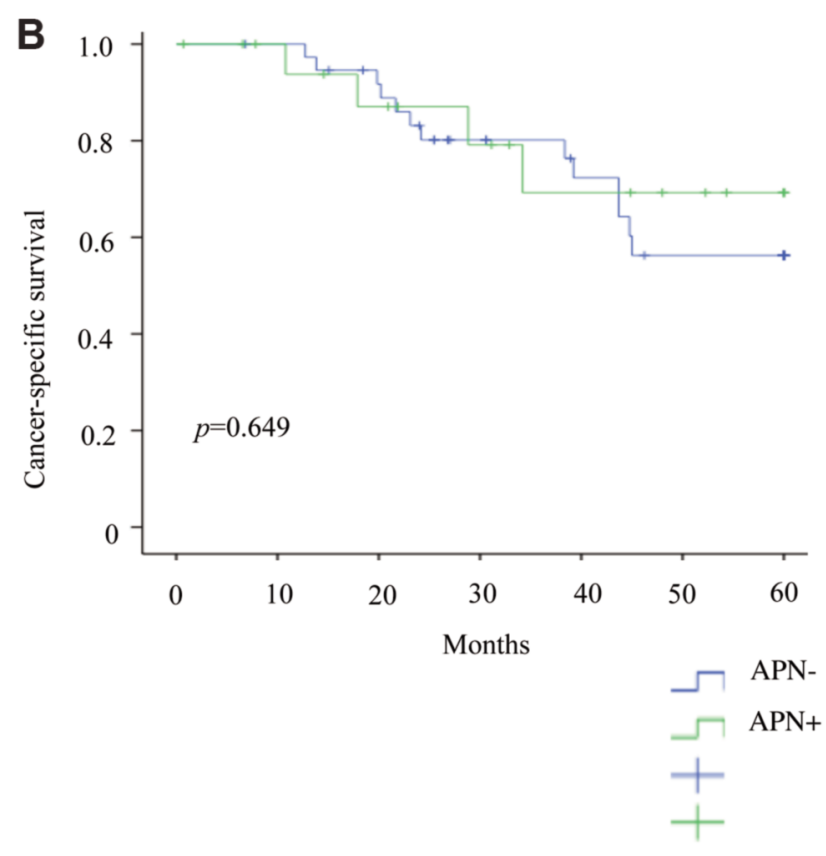

Figure 6. Patient outcomes after matching: (A) Relapse-free survival and $(B)$ cancer-specific survival.

$p<0.001$ ), as well as significantly higher rates of nodal positivity $(0.342 \pm 0.042$ vs. $0.125 \pm 0.004 ; p<0.001)$, postoperative recurrence $(57.89 \%$ vs. $26.51 \% ; p=0.002)$, para-aortic lymph node involvement (15.78\% vs. $2.08 \%$; $p<0.001)$, and lung $(26.31 \%$ vs. $8.35 \% ; p=0.036)$ metastasis (Table IV). After PSM, the APN+ (vs. APN-) group continued to show more intraoperative bleeding and nodal metastases, whereas rates of local recurrence, distant metastasis, and overall recurrence did not differ significantly (Table IV).

\section{Discussion}

Through this study, we determined that the correlation of APN metastases with other adverse features suggests it is an indicator of an underlying biological propensity for distant metastases rather than the origin of distant metastases. Although APN+ patients often show deeper tumor infiltration, greater lymphatic involvement, and more nodal metastases at diagnosis, postoperative relapse is the consequence. Once all baselines are leveled, APN+ status 
Table II. Cox regression analysis of patient prognosis prior to matching.

\begin{tabular}{|c|c|c|c|c|c|c|}
\hline \multirow[t]{2}{*}{ Parameters } & \multicolumn{3}{|c|}{ Relapse-free survival } & \multicolumn{3}{|c|}{ Cancer-specific survival } \\
\hline & HR & $\mathrm{HR}(95 \% \mathrm{CI})$ & $p$-Value & HR & HR $(95 \%$ CI $)$ & $p$-Value \\
\hline Apical node metastasis, Yes/No & 0.777 & $0.372-1.62$ & 0.5 & 1.347 & $0.391-4.643$ & 0.637 \\
\hline Gender, M/F & 1.3 & $0.89-1.899$ & 0.175 & 1.187 & $0.646-2.181$ & 0.581 \\
\hline Age, years & 0.997 & $0.979-1.014$ & 0.709 & 1.019 & $0.99-1.049$ & 0.201 \\
\hline Postoperative complications, Yes/No & 0.835 & $0.548-1.272$ & 0.401 & 0.459 & $0.25-0.845$ & 0.012 \\
\hline CEA level, $\mathrm{ng} / \mathrm{ml} \geq 5 /<5$ & 0.669 & $0.472-0.948$ & 0.024 & 0.505 & $0.293-0.87$ & 0.014 \\
\hline MUC, SRC, Poor vs. ADC & 0.568 & $0.275-1.171$ & 0.125 & 0.221 & $0.08-0.609$ & 0.004 \\
\hline Perineural infiltration, Yes/No & 0.581 & $0.34-0.992$ & 0.047 & 0.927 & $0.355-2.42$ & 0.876 \\
\hline Lymphatic involvement, Yes/No & 1.072 & $0.74-1.553$ & 0.713 & 0.718 & $0.396-1.302$ & 0.275 \\
\hline Vascular invasion, Yes/No & 0.984 & $0.632-1.531$ & 0.942 & 1.164 & $0.573-2.365$ & 0.674 \\
\hline Tumor-positive node count & 1.112 & $1.042-1.187$ & 0.001 & 1.181 & $1.076-1.297$ & $<0.001$ \\
\hline Harvested lymph node total & 0.982 & $0.966-0.999$ & 0.038 & 0.981 & $0.955-1.009$ & 0.179 \\
\hline Infiltrative depth, T1-2/T3-4 & 0.348 & $0.172-0.706$ & 0.003 & 0.215 & $0.049-0.946$ & 0.042 \\
\hline Nodal metastasis, N1/N2 & 0.787 & $0.492-1.26$ & 0.319 & 0.759 & $0.37-1.558$ & 0.452 \\
\hline
\end{tabular}

ADC, Adenocarcinoma; CEA, carcinoembryonic antigen; CI, confidence interval; HR, hazard ratio; MUC, mucinous adenocarcinoma; Poor, poorly differentiated adenocarcinoma; SRC, signet-ring cell carcinoma.

Table III. Cox regression analysis of patient after matching.

\begin{tabular}{|c|c|c|c|c|c|c|}
\hline \multirow[t]{2}{*}{ Parameters } & \multicolumn{3}{|c|}{ Relapse-free survival } & \multicolumn{3}{|c|}{ Cancer-specific survival } \\
\hline & HR & HR $(95 \%$ CI $)$ & $p$-Value & $\mathrm{HR}$ & HR $(95 \% \mathrm{CI})$ & $p$-Value \\
\hline Apical node metastasis, Yes/No & 0.627 & $0.221-1.776$ & 0.379 & 273.5 & $0.129-5.780$ & 0.151 \\
\hline Gender, M/F & 0.59 & $0.168-2.078$ & 0.412 & 19.204 & $0.302-1219.7$ & 0.163 \\
\hline Age, years & 1.017 & $0.961-1.076$ & 0.564 & 2.149 & $1.249-3.696$ & 0.006 \\
\hline Postoperative complications, Yes/No & 2.849 & $0.975-8.325$ & 0.056 & 601.2 & $3.219-11227$ & 0.016 \\
\hline CEA level, $\mathrm{ng} / \mathrm{ml} \geq 5 /<5$ & 0.446 & $0.157-1.269$ & 0.013 & 0.46 & $0.02-10.355$ & 0.01 \\
\hline MUC, SRC, Poor vs. ADC & 0.049 & $0.002-1.399$ & 0.078 & 0.001 & $0-1.567$ & 0.059 \\
\hline Perineural infiltration, Yes/No & 0.568 & $0.121-2.669$ & 0.473 & 0.001 & $0.112-0.021$ & 0.979 \\
\hline Lymphatic involvement, Yes/No & 0.273 & $0.062-1.209$ & 0.087 & 5855 & $0.741-46267$ & 0.058 \\
\hline Vascular invasion, Yes/No & 0.76 & $0.074-7.776$ & 0.817 & 0.001 & $0-1.638$ & 0.066 \\
\hline Tumor-positive node count & 1.005 & $0.89-1.136$ & 0.934 & 3.592 & $1.186-10.876$ & 0.024 \\
\hline Harvested lymph node total & 1.01 & $0.962-1.06$ & 0.689 & 1.223 & $0.885-1.69$ & 0.222 \\
\hline Infiltrative depth, T1-2/T3-4 & 8.011 & $1.589-40.393$ & 0.012 & 0.419 & $0.003-50.734$ & 0.722 \\
\hline Nodal metastasis, N1/N2 & 0.763 & $0.152-3.833$ & 0.743 & 0.001 & $0-3.21$ & 0.828 \\
\hline
\end{tabular}

ADC, Adenocarcinoma; CEA, carcinoembryonic antigen; CI, confidence interval; HR, hazard ratio; MUC, mucinous adenocarcinoma; Poor, poorly differentiated adenocarcinoma; SRC, signet-ring cell carcinoma.

appears unrelated to local tumor recurrence or poorer longterm survival. APN metastasis may thus be considered a regional rather than systemic manifestation.

The analysis we performed before PSM generated outcomes readily reflected in current research publications (4, 7-10). However, results obtained after PSM were also corroborated by certain reports $(1,11)$, creating stark contradictions. The findings were nonetheless quite explainable. By adopting a sex-matched scoring method, applied to the maximum extent feasible, baseline differences between groups were effectively eliminated.
In our results, it was also apparent that as nodal metastasis approached the root of IMA, prognosis worsened. On the other hand, many studies support the premise that APN+ status has little prognostic impact in the setting of CRC. The chief reason for these conflicting views is perhaps rooted in the definition of APN. Western world defines the APN region as an area $\sim 1 \mathrm{~cm}$ from IMA origination (14). In the Japanese classification system, the APN region extends from the origin of IMA to the branching of left colic artery (LCA) (3). By this definition, the APN region often exceeds $1 \mathrm{~cm}$ and is more open to harvesting. 
Wang et al: Apical Lymph Node Metastasis Due to Left-Sided Colorectal Cancer

Table IV. Nodal involvement, surgical metrics, and postoperative recurrences before and after matching.

\begin{tabular}{|c|c|c|c|c|c|c|}
\hline \multirow[t]{2}{*}{ Parameters } & \multicolumn{3}{|c|}{ Before matching } & \multicolumn{3}{|c|}{ After matching } \\
\hline & APN- $(n=479)$ & $\mathrm{APN}+(\mathrm{n}=19)$ & $p$-Value & APN- $(n=38)$ & $\mathrm{APN}+(\mathrm{n}=19)$ & $p$-Value \\
\hline Harvested lymph node total & $26.41 \pm 0.51$ & $27.98 \pm 2.80$ & N.S. & $29.89 \pm 2.03$ & $27.98 \pm 2.80$ & N.S. \\
\hline Tumor-positive node count & $2.98 \pm 0.12$ & $9.58 \pm 1.64$ & $<0.001$ & $6.03 \pm 0.586$ & $9.58 \pm 1.64$ & 0.015 \\
\hline Nodal tumor-positivity rate & $0.125 \pm 0.004$ & $0.342 \pm 0.042$ & $<0.001$ & $0.228 \pm 0.024$ & $0.342 \pm 0.042$ & N.S. \\
\hline Operative time & $220.47 \pm 3.55$ & $241.78 \pm 20.34$ & N.S. & $246.55 \pm 12.85$ & $241.78 \pm 20.34$ & N.S. \\
\hline Surgical bleeding (ml) & $48.43 \pm 5.95$ & $134.53 \pm 38.47$ & 0.006 & $60.37 \pm 19.7$ & $134.53 \pm 38.47$ & 0.015 \\
\hline $\mathrm{pRM}$ & $20(4.2)$ & $5(26.3)$ & 0.002 & $1(2.6)$ & $5(26.3)$ & 0.013 \\
\hline Total recurrences & $127(26.51)$ & $11(57.89)$ & 0.002 & $23(60.52)$ & $11(57.89)$ & N.S. \\
\hline Para-aortic nodes & $10(2.08)$ & $3(15.78)$ & $<0.001$ & $5(13.15)$ & $3(15.78)$ & N.S. \\
\hline Local & $21(4.38)$ & $0(0.0)$ & N.S. & $6(15.78)$ & $0(0.0)$ & 0.067 \\
\hline Liver & $57(11.89)$ & $5(26.31)$ & N.S. & $11(28.94)$ & $5(26.31)$ & N.S. \\
\hline Lung & $40(8.35)$ & $5(26.31)$ & 0.036 & $4(10.52)$ & $5(26.31)$ & N.S. \\
\hline Peritoneal & $9(1.87)$ & $0(0.0)$ & N.S. & $4(10.52)$ & $0(0.0)$ & N.S. \\
\hline Other sites & $3(0.62)$ & $1(5.26)$ & N.S. & $4(10.52)$ & $1(5.26)$ & N.S. \\
\hline
\end{tabular}

APN-, Tumor-negative apical lymph nodes; APN+, tumor-positive apical lymph nodes; pRM, pathologic resection margin.

During left-sided colorectal apical lymphadenectomy, LCA retention has been a contentious issue (14-17). Some sources have suggested that high-level ligation of IMA may cause postoperative urinary dysfunction, despite an understanding of hypogastric nerve anatomy and the benefit of endoscopic surgical magnification. The reduction in blood supply may also increase the risk of anastomotic leakage $(7,18)$. LCA was not spared in the vast majority of our patients, allowing us to adequately remove lymphatics of the vascular sheath at the IMA root (19). Yet, this did not increase the risk of anastomotic leakage or urinary disturbance in our patients (data not shown). IMA root dissection contributed to the average yields 26-27 lymph nodes, affording more accurate postoperative CRC staging (20).

Para-aortic nodal involvement and distant tumor metastasis were clearly more common in APN+ (vs. APN-) patients (4). Positive para-aortic nodes were confirmed in $15.78 \%$ of APN+ group members, so for those patients with deeper tumors (T3/T4) or elevated preoperative CEA levels, close follow-up surveillance of para-aortic lymph nodes is essential. As a future endeavor, a prospective multicenter clinical trial assessing prophylactic dissection of lymph nodes around the aorta in IMA APN+ patients might be worthwhile.

Increasingly, more attention has been paid to the circumferential margin (pRM) of rectal cancer $(21,22)$. We identified five APN+ patients (26.3\%) with pRM tumor positivity, far surpassing the number of patients similarly affected in the APN- group. Although pRM involvement did not surface as a prognostic risk factor in multivariate analysis, close monitoring for potential local recurrence is warranted, given the limited number of patients we studied.
Traditionally, the pathological characteristics of cancer cells are generally regarded to play an important role in the progression of lymph node metastasis to systemic metastasis. Therefore, APNs are considered to be "the last line of defense against metastatic disease". However, reported cases of systemic metastases without lymph node metastasis break these traditional concepts. Therefore, instead of focusing on the presence or absence of apical lymph nodes, we should pay more attention to their pathological background.

Although the literature indicates that APN+ patients tend to have a poor prognosis (9), there was no evidence in the current analysis to support APN as an independent risk factor. However, the number of positive lymph node metastases was an independent risk factor for CSS both before and after PSM. Therefore, the current TNM staging based on the number of metastatic lymph nodes seems to be more reasonable than the regional lymph node classification method.

The decision to perform a routine D3 dissection with the removal of the apical nodes require continued review. According to our data, only $3.8 \%$ of patients with lymph node metastasis have APN+ cancer. Should surgeons routinely remove apical lymph nodes, even if the rate of metastasis is so low (19)? Intuitively, all surgeons know that the possible benefits will be very small (if any). In patients with micrometastasis, a positive APN that is not removed may result in lymph node recurrence around the para-aortic region. Therefore, from the perspective of radical resection, conventional apical lymph node excision may be beneficial. In the Japanese guidelines for colorectal cancer, in view of the low metastasis of IMA apical lymph nodes, D2 lymph node dissection is sufficient for T1 infiltrating colorectal cancer. But for tumors infiltrating 
more than $\mathrm{T} 2$, routine $\mathrm{D} 3$ lymph node dissection is recommended.

This study has certain acknowledged shortcomings. The number APN+ patients were still limited despite a 10-year period. In addition, we were forced to sacrifice a substantial volume of patient information through PSM-enabled masking of group bias. Finally, a proper analysis of postoperative chemotherapy treatment was prohibited by individual differences in treatment regimens. This may have impacted our results to a degree.

\section{Conclusion}

According to our findings, APN positivity may constitute a regional rather than systemic manifestation. The TNM staging based on the number of metastatic lymph nodes seems to be more reasonable than the regional lymph node classification method.

\section{Conflicts of Interest}

The Authors have no conflicts of interest to declare in terms of this study.

\section{Authors' Contributions}

LMW drafted the manuscript. YH, GH, TI, HK, KH, NO, MA and SY reviewed its content. All Authors have read and approved the final submission.

\section{Acknowledgements}

The Authors would like to thank BioMed Proofreading, LLC for English copyediting.

\section{References}

1 Yeom SS, Lee SY, Kim CH, Kim HR and Kim YJ: The prognostic effect of adjuvant chemotherapy in the colon cancer patients with solitary lymph node metastasis. Int J Colorectal Dis 34(8): 1483-1490, 2019. PMID: 31292725. DOI: 10.1007/ s00384-019-03346-7

2 Hashiguchi Y, Muro K, Saito Y, Ito Y, Ajioka Y, Hamaguchi T, Hasegawa $K$, Hotta $K$, Ishida $H$, Ishiguro $M$, Ishihara $S$, Kanemitsu Y, Kinugasa Y, Murofushi K, Nakajima TE, Oka S, Tanaka T, Taniguchi H, Tsuji A, Uehara K, Ueno H, Yamanaka T, Yamazaki K, Yoshida M, Yoshino T, Itabashi M, Sakamaki K, Sano K, Shimada Y, Tanaka S, Uetake H, Yamaguchi S, Yamaguchi N, Kobayashi H, Matsuda K, Kotake K, Sugihara K and Japanese Society for Cancer of the Colon and Rectum: Japanese society for cancer of the colon and rectum (jsccr) guidelines 2019 for the treatment of colorectal cancer. Int J Clin Oncol 25(1): 1-42, 2020. PMID: 31203527. DOI: 10.1007/ s10147-019-01485-z

3 Japanese Society for Cancer of the Colon and Rectum: Japanese classification of colorectal, appendiceal, and anal carcinoma:
The 3d english edition [secondary publication]. J Anus Rectum Colon 3(4): 175-195, 2019. PMID: 31768468. DOI: 10.23922/ jarc.2019-018

4 Tsai HL, Chen YT, Yeh YS, Huang CW, Ma CJ and Wang JY: Apical lymph nodes in the distant metastases and prognosis of patients with stage iii colorectal cancer with adequate lymph node retrieval following folfox adjuvant chemotherapy. Pathol Oncol Res 25(3): 905-913, 2019. PMID: 29299827. DOI: 10.1007/s12253-017-0381-5

5 Kawada H, Kurita N, Nakamura F, Kawamura J, Hasegawa S, Kotake K, Sugihara K, Fukuhara S and Sakai Y: Incorporation of apical lymph node status into the seventh edition of the tnm classification improves prediction of prognosis in stage iii colonic cancer. Br J Surg 101(9): 1143-1152, 2014. PMID: 24947893. DOI: $10.1002 /$ bjs. 9548

6 Kang J, Hur H, Min BS, Kim NK and Lee KY: Prognostic impact of inferior mesenteric artery lymph node metastasis in colorectal cancer. Ann Surg Oncol 18(3): 704-710, 2011. PMID: 20857225. DOI: 10.1245/s10434-010-1291-x

7 Ang CW, Tweedle EM, Campbell F and Rooney PS: Apical node metastasis independently predicts poor survival in dukes $\mathrm{c}$ colorectal cancer. Colorectal Dis 13(5): 526-531, 2011. PMID: 20070342. DOI: 10.1111/j.1463-1318.2010.02188.x

8 Malassagne B, Valleur P, Serra J, Sarnacki S, Galian A, Hoang $\mathrm{C}$ and Hautefeuille P: Relationship of apical lymph node involvement to survival in resected colon carcinoma. Dis Colon Rectum 36(7): 645-653, 1993. PMID: 8394236. DOI: $10.1007 / \mathrm{bf02238591}$

9 Huh JW, Kim YJ and Kim HR: Distribution of lymph node metastases is an independent predictor of survival for sigmoid colon and rectal cancer. Ann Surg 255(1): 70-78, 2012. PMID: 22133895. DOI: 10.1097/SLA.0b013e31823785f6

10 Peng J, Wu H, Li X, Sheng W, Huang D, Guan Z, Wang M and Cai S: Prognostic significance of apical lymph node metastasis in patients with node-positive rectal cancer. Colorectal Dis 15(1): e13-20, 2013. PMID: 23057847. DOI: 10.1111/codi. 12055

11 Yi JW, Lee TG, Lee HS, Heo SC, Jeong SY, Park KJ and Kang $\mathrm{SB}$ : Apical-node metastasis in sigmoid colon or rectal cancer: Is it a factor that indicates a poor prognosis after high ligation? Int J Colorectal Dis 27(1): 81-87, 2012. PMID: 21739197. DOI: $10.1007 / \mathrm{s} 00384-011-1271-\mathrm{z}$

12 Wang L, Hirano Y, Heng G, Ishii T, Kondo H, Hara K, Obara N, Asari M, Kato T and Yamaguchi S: Mucinous adenocarcinoma as a high-risk factor in stage II colorectal cancer: A propensity score-matched study from japan. Anticancer Res 40(3): 16511659, 2020. PMID: 32132070. DOI: 10.21873/anticanres.14115

13 Sakamoto Y, Miyamoto Y, Tokunaga R, Akiyama T, Daitoku N, Hiyoshi Y, Iwatsuki M, Baba Y, Iwagami S, Yoshida N and Baba $\mathrm{H}$ : Long-term outcomes of colorectal cancer surgery for elderly patients: A propensity score-matched analysis. Surg Today, 2019. PMID: 31844988. DOI: 10.1007/s00595-019-01934-2

14 Yang X, Ma P, Zhang X, Wei M, He Y, Gu C, Deng X and Wang $\mathrm{Z}$ : Preservation versus non-preservation of left colic artery in colorectal cancer surgery: An updated systematic review and meta-analysis. Medicine (Baltimore) 98(5): e13720, 2019. PMID: 30702552. DOI: 10.1097/MD.0000000000013720

15 Maeda Y, Shinohara T, Futakawa N, Minagawa N, Sunahara M, Koyama R, Nagatsu A and Hamada T: The oncologic outcomes of inferior mesenteric artery-preserving laparoscopic lymph node 
dissection for upper-rectal or sigmoid colon cancer. J Laparoendosc Adv Surg Tech A 28(11): 1352-1358, 2018. PMID: 30192168. DOI: 10.1089/lap.2018.0201

16 Guo Y, Wang D, He L, Zhang Y, Zhao S, Zhang L, Sun X and Suo J: Marginal artery stump pressure in left colic arterypreserving rectal cancer surgery: A clinical trial. ANZ J Surg 87(7-8): 576-581, 2017. PMID: 25708562. DOI: 10.1111/ ans. 13032

17 Kobayashi M, Okamoto K, Namikawa T, Okabayashi T and Araki K: Laparoscopic lymph node dissection around the inferior mesenteric artery for cancer in the lower sigmoid colon and rectum: Is d3 lymph node dissection with preservation of the left colic artery feasible? Surg Endosc 20(4): 563-569, 2006. PMID: 16391959. DOI: 10.1007/s00464-005-0160-3

18 Inomata $\mathrm{M}$, Shiroshita H, Uchida $\mathrm{H}$, Bandoh T, Akira S, Yamaguchi S, Kurokawa Y, Seki Y, Eguchi S, Wada N, Takiguchi S, Ieiri S, Endo S, Iwazaki M, Sato Y, Tamaki Y, Kitamura K, Tabata M, Kanayama H, Mimata H, Hasegawa T, Takahashi H, Onishi K, Uemura T, Hashizume M, Matsumoto $\mathrm{S}$, Kitano $\mathrm{S}$ and Watanabe M: Current status of endoscopic surgery in japan: The 14th national survey of endoscopic surgery by the japan society for endoscopic surgery. Asian J Endosc Surg 13(1): 7-18, 2020. PMID: 31828925. DOI: 10.1111/ases.12768

19 Alici A, Kement M, Gezen C, Akin T, Vural S, Okkabaz N, Basturk E, Yegenoglu A and Oncel M: Apical lymph nodes at the root of the inferior mesenteric artery in distal colorectal cancer: An analysis of the risk of tumor involvement and the impact of high ligation on anastomotic integrity. Tech Coloproctol 14(1): 1-8, 2010. PMID: 20066459. DOI: 10.1007/ s10151-009-0547-6
20 Kwon TS, Choi SB, Lee YS, Kim JG, Oh ST and Lee IK: Novel methods of lymph node evaluation for predicting the prognosis of colorectal cancer patients with inadequate lymph node harvest. Cancer Res Treat 48(1): 216-224, 2016. PMID: 25943323. DOI: 10.4143/crt.2014.312

21 Han C, Yan P, Jing W, Li M, Du B, Si M, Yang J, Yang K, Cai $\mathrm{H}$ and Guo T: Clinical, pathological, and oncologic outcomes of robotic-assisted versus laparoscopic proctectomy for rectal cancer: A meta-analysis of randomized controlled studies. Asian J Surg, 2020. PMID: 31964585. DOI: 10.1016/j.asjsur.2019. 11.003

22 Compton C, Fenoglio-Preiser CM, Pettigrew N and Fielding LP: American joint committee on cancer prognostic factors consensus conference: Colorectal working group. Cancer $88(7)$ : 1739-1757, 2000. PMID: 10738234. DOI: 10.1002/(sici)10970142(20000401)88:7<1739::aid-cncr30>3.0.co;2-t

Received June 15, 2020

Revised June 30, 2020

Accepted July 6, 2020 